\title{
Commentary \\ Distinct functions of BRCA1 and BRCA2 in double-strand break repair
}

\author{
Yilun Liu and Stephen C West
}

Imperial Cancer Research Fund, Clare Hall Laboratories, South Mimms, Hertfordshire, UK

Correspondence: Stephen C West, Imperial Cancer Research Fund, Clare Hall Laboratories, Blanche Lane, South Mimms, Hertfordshire, EN6 3LD, UK. Tel: +44 0207269 3868; fax: +44 0207269 3811; e-mail: s.west@icrf.icnet.uk

Received: 6 September 2001

Breast Cancer Res 2002, 4:9-13

Revisions requested: 24 September 2001

Revisions received: 5 October 2001

Accepted: 12 October 2001

Published: 20 November 2001

(C) 2002 BioMed Central Ltd

(Print ISSN 1465-5411; Online ISSN 1465-542X)

\begin{abstract}
Individuals carrying BRCA mutations are predisposed to breast cancer. The BRCA1 and BRCA2 proteins are required for homologous recombination and DNA break repair, leading to the suggestion that they act in concert. However, direct evidence of a stable BRCA1/BRCA2 complex has not been demonstrated. Rather, the two proteins have been found as constituents of discrete, but perhaps nonexclusive complexes that are critical for repair. We discuss the interaction of BRCA1 with the $\mathrm{BACH} 1$ and BARD1 proteins, and suggest that the pleiotropic nature of mutations in BRCA1 may be associated with defects in protein-protein interactions In contrast, the role of BRCA2 in DNA repair may be more defined by its direct interaction with the RAD51 recombinase.
\end{abstract}

Keywords: BRCA1, BRCA2, DNA repair, homologous recombination, RAD51

\section{Introduction}

Almost 5\% of individuals with breast cancers are predisposed to the disease by genetic mutations. The discovery of the BRCA1 and BRCA2 genes, and of how mutations in these genes lead to the onset of breast and ovarian cancers has provided new insights into the molecular mechanisms that lead to tumourigenesis and have improved our understanding of cancer predisposition [1-3]. In current research the roles of BRCA1 and BRCA2 are beginning to be unravelled, and it is hoped that identification of the precise properties of BRCA1 and BRCA2 will provide an important key to cancer prevention and development of new cancer treatments.

Both BRCA1 and BRCA2 are essential genes for cellular development; deletions in either gene lead to embryonic lethality in mice [4-8]. Genetic studies conducted in BRCA1- and BRCA2-defective cell lines $[7,9,10]$ have further revealed that these tumour suppressor genes are required for maintenance of genome integrity and for normal levels of resistance to DNA damage. Moreover, without functional BRCA genes, cells are inefficient in repairing DNA damage by homologous recombination [11-14], which can lead to apoptosis or cell transformation $[15,16]$.

Early studies on BRCA1 (208 kDa) and BRCA2 (384 $\mathrm{kDa}$ ) led to the conclusion that the two proteins function together as part of a complex that acts directly in doublestrand break (DSB) repair. First, BRCA1- and BRCA2defective cell lines and knockout mice exhibit similar sensitivities to DNA-damaging agents $[4,16]$. Second, $B R C A 1$ - and BRCA2-defective cell lines show defects in DSB repair by homologous recombination $[12,14]$. Third, the BRCA1 and BRCA2 proteins co-localize to DNAdamage-induced nuclear foci, and to foci that appear in $S$ phase [17]. Even though BRCA1 and BRCA2 are able to interact with each other, however, only a minority of the

$\mathrm{DSB}=$ double-strand break; NHEJ = nonhomologous end joining. 
BRCA1 was actually found in association with BRCA2 $[17,18]$. Moreover, the recent identification of proteins that associate with either BRCA1 or BRCA2 indicates that the two BRCA proteins each participate in different protein complexes, and that these complexes may play quite distinct roles in DSB repair.

A summary of some of the interactions that have been described for BRCA1 is shown in Fig. 1. In brief, BRCA1 has been shown to be part of a genome surveillance complex termed BASC, which contains a number of proteins that are important for efficient DNA repair (the MRE11/RAD50/NBS1 repair complex, proteins involved in mismatch repair such as the MSH2/MSH6 and PMS2/ MLH1 heterodimers, the DNA-damage-signalling protein kinase ATM, BLM helicase and replication factor-C) [18]. Additionally, BRCA1 associates with RNA polymerase II holoenzyme, the cell-cycle-checkpoint kinase ATR, a SWI/SNF chromatin-remodelling complex, the Fanconi's anaemia protein FANCD2, and a number of other important proteins (for review $[19,20]$ ). In many cases, however, the specificity and significance of these protein/protein interactions may require further investigation. The interactions of BRCA2 appear more limited, with the most illuminating interaction being that of BRCA2 with the essential homologous recombination/repair protein RAD51 [21].

In the present commentary we focus our attention on a putative DNA helicase termed $\mathrm{BACH} 1$, which was recently shown to interact with BRCA1, and on another BRCA1-interacting protein known as BARD1 [22-24]. By comparing various aspects of the BRCA1/BARD1/ BACH1 complex and the BRCA2/RAD51 complex, we attempt to account for the related repair-defective phenotypes associated with $B R C A 1$ and BRCA2 mutations.

\section{Regulation of BACH1 functions in DNA repair by BRCA1}

The carboxyl-terminal region of BRCA1 contains two BRCT motifs, which are important for protein/protein interactions [25]. Interestingly, many cancer-associated mutations in BRCA1 result in the formation of a truncated BRCA1 protein, in which the carboxyl-terminal region has been deleted. It is therefore possible that proteins that interact with the BRCT motifs of BRCA1 may play important roles in BRCA1-dependent tumour suppression.

Recently, Cantor et al. [24] identified a novel 130-kDa polypeptide on the basis of its interaction with the BRCT motifs of BRCA1. Analysis of the sequence revealed that the protein contained the seven motifs that are characteristic of the DEAH helicase family, leading to the name BACH1 (BRCA1-associated carboxyl-terminal helicase). However, intrinsic DNA helicase activity has not yet been demonstrated. The association of BRCA1 with BACH1 was confirmed by three independent methods:
Figure 1

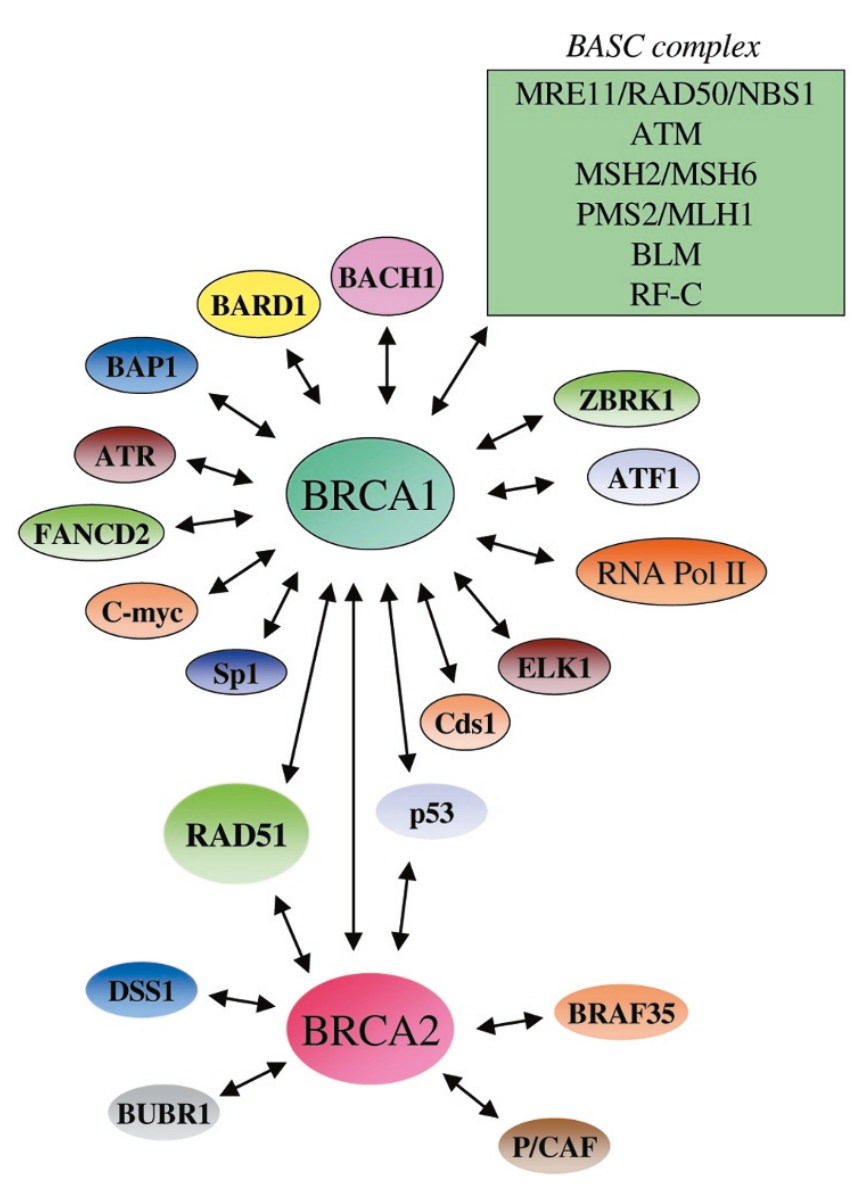

Interactions of BRCA1 and BRCA2 with other proteins, or protein complexes. as determined by two-hybrid screens, co-immunoprecipitation analyses and co-fractionation studies. In general, the protein-protein associations of BRCA1 have been shown to be distinct from those exhibited by BRCA2.

GST-BRCA1 fusion proteins were used to affinity purify $\mathrm{BACH} 1$ from whole-cell extracts; BRCA1 and BACH1 were co-immunoprecipitated from MCF-7 cells using either anti-BRCT antibodies or anti-BACH1 antibodies; and BRCA1 and BACH1 were shown to co-localize to nuclear foci in $S$ and $G 2$ phase cells by immunofluorescence microscopy. Most importantly, functional BRCA1 was shown to be essential for nuclear focus formation by $\mathrm{BACH} 1$, suggesting that BRCA1 plays a role in regulating the cellular localization of $\mathrm{BACH} 1$.

BRCA1 is potentially involved in transcription, replication and DNA repair, and it is therefore possible that the putative helicase $\mathrm{BACH} 1$ could be involved in any of these cellular processes. In order to address the significance of $\mathrm{BACH} 1$ in DSB repair, Cantor et al. [24] showed that over-expression of a $B A C H 1$ gene carrying a mutation in 
the sequence encoding the conserved lysine residue (Lys52 $\rightarrow$ Arg) in the ATPase domain greatly decreased the ability of the cells to repair DSBs promptly [24]. Interestingly, this dominant-negative phenotype was rescued by over-expression of $\mathrm{BACH} 1$ carrying a second mutation that abolished interactions between the mutant $\mathrm{BACH} 1$ and BRCA1. Those findings show that the repair function of $\mathrm{BACH} 1$ is mediated through its interactions with BRCA1. It is possible that BRCA1 is required for the specific recruitment of $\mathrm{BACH} 1$ to the sites of repair.

$\mathrm{BACH} 1$ mutations have been identified in two out of 65 women with early-onset breast cancer [24], one of whom had a familial history of breast and ovarian cancer. Both individuals were normal for BRCA1 and BRCA2. One mutation (Pro47 $\rightarrow \mathrm{Ala}$ ), in the highly conserved ATPbinding domain, was found to lead to destabilization of $\mathrm{BACH} 1$ protein. Clearly, this mutation will result in loss of function of $\mathrm{BACH} 1$. The second mutation (Met299 $\rightarrow$ le) is a conservative substitution in a nonconserved residue, and its functional significance is unclear. Unfortunately, the correlation of these mutations with DSB repair efficiency and genomic instability was not determined.

\section{Additional functions of BRCA1 in DNA repair indicated by interactions with BARD1}

Another protein that is known to interact with the BRCA1/BACH1 complex is BARD1 [24]. First isolated in a two-hybrid screen for BRCA1-interacting proteins, both BARD1 and BRCA1 contain a conserved RING finger motif, which is often linked to either DNA binding or ubiquitination [22]. The RING finger motif of BRCA1 has been shown to possess ubiquitin ligase activity, which is greatly stimulated by binding to BARD1 $[23,26]$. Significantly, a breast-cancer-associated $B R C A 1$ mutation within the RING finger motif abolished the ubiquitin ligase activity of BRCA1, indicating that this activity is important for the action of BRCA1 in tumour suppression [26]. Moreover, several mutations located in the RING finger motif of BRCA1, which disrupt the ubiquitin ligase activity, have also been shown to cause increased sensitivity to DNAdamaging agents. This correlation suggests an additional role for BRCA1 in repair of DNA damage, in addition to its role as a regulator for $\mathrm{BACH} 1$, in targeting protein(s) for degradation.

Interestingly, BRCA1 also interacts with BAP1, a ubiquitin hydrolase [27]. Because BAP1 and BARD1 can modulate ubiquitination by BRCA1, and both bind the RING motif of BRCA1, there appears to be an intriguing interplay between these proteins in terms of the promotion (BARD1) or prevention (BAP1) of ubiquitin-mediated protein degradation.

A likely target for the ubiquitin ligase activity of BRCA1 is the Fanconi anaemia protein FANCD2, which is known to be mono-ubiquitinated in a BRCA1-dependent manner in response to DNA damage [28]. Consistent with this proposal, FANCD2 co-localizes with BRCA1 in DNAdamage-induced nuclear foci, FANCD2 and BRCA1 can be co-immunoprecipitated together, and mutations in FANCD2 result in hypersensitivity to various DNA-damaging agents [28].

\section{Distinct roles for BRCA1/BACH1 and BRCA2/RAD51 complexes in repair of double-strand breaks}

Because BRCA1 interacts with a wide range of proteins, it is likely that the protein plays a multifunctional role in DNA metabolism. Two such roles may be defined by its interactions with $\mathrm{BACH} 1$ and with BARD1, both of which impact on the efficiency of DNA repair (Fig. 2). These functions may also require phosphorylation of BRCA 1 by the protein kinase ATM in response to DNA damage [7]. What, then, are the precise functions of the BRCA1/BACH1/BARD1 complex in relation to recombinational repair? It is interesting that several of the proteins that interact with BRCA1 exhibit helicase/nuclease activities (e.g. the BACH1, BLM, MRE11/RAD50/NBS1 complex) and it is possible that BRCA1 may assist the targeting of these proteins to DSBs, where they play a role in break resection. Alternatively, proteins that interact with BRCA1 may be involved in the processing of recombination intermediates that contain Holliday junctions. Support for these potential roles comes from observations indicating that BRCA1 binds DNA and inhibits the exonuclease activities of the MRE11/RAD50/NBS1 complex, and that it also displays a preference for branched DNA structures [29]. The ubiquitin ligase activity of the BRCA1/BACH1/BARD1 complex might be important for down-regulating proteins that are involved in cell/cycle progression during the initial step of DNA repair, or DNA recombination proteins after the repair process is completed.

Like cells that are defective in $B R C A 1, B R C A 2$-defective cells are also defective in homologous recombination [14], leading to the repair of DSBs by alternative mechanisms that involve nonhomologous end joining (NHEJ) $[9,30]$. The consequences of NHEJ can be disastrous for the cell because, unlike the conservative process of homologous recombination, NHEJ can occur between breaks on different chromosomes, resulting in chromosomal translocations or deletions. Such changes can lead to improper regulation of gene expression that could, in turn, result in cell transformation and cancer development.

BRCA2 protein interacts directly with the RAD51 protein, the cellular recombinase that is essential for the initiation of DNA/DNA interactions that lead to strand exchange [31]. The ease with which these two proteins can be coimmunoprecipitated from cell-free extracts [17] indicates that BRCA2 may play a more direct role in DNA repair than BRCA1 (Fig. 2). RAD51 interacts with BRCA2 by 
Figure 2

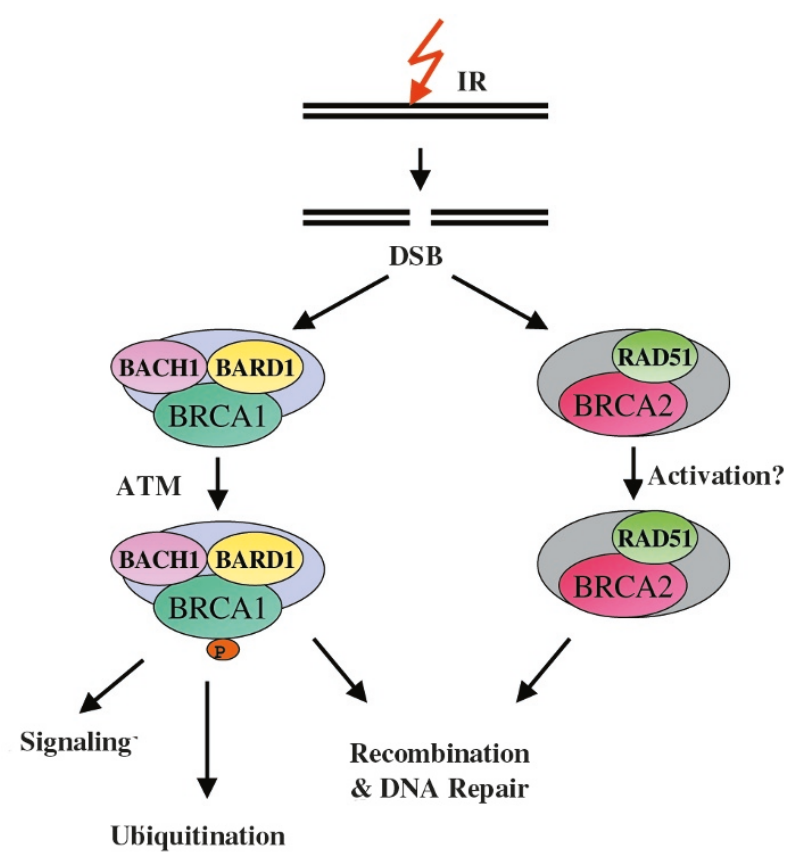

Distinct complexes formed by BRCA1 and BRCA2 are involved in a variety of cellular processes such as damage signalling, protein degradation and both direct and indirect effects on the mechanisms of homologous recombination and DNA repair. Defects in these processes can lead to inefficient repair of DNA damage, genomic instability and tumourigenesis.

binding to a series of BRC repeats that are present in BRCA2 [21,32]. In recent in vitro studies [33], it was shown that peptides that correspond to the BRC3 or BRC4 repeats of BRCA2 bind RAD51 and block its ability to form nucleoprotein filaments on DNA. This observation helps to explain the results of earlier studies conducted by Chen et al. [34], in which it was shown that over-expression of the BRC4 repeat in mammalian cells resulted in a hypersensitivity to DNA-damaging agents. Presumably, high levels of the BRC4 repeat competed with BRCA2 for RAD51, resulting in a dominant-negative phenotype. Because DNA binding and nucleoprotein filament formation by RAD51 is required for all recombination activities exhibited by the protein, those observations indicate that BRCA2 plays an important regulatory role with respect to RAD51. Those studies also showed that mutations within the $\mathrm{BRC}$ region that mimic cancer-associated BRCA2 mutations failed to inhibit DNA binding by RAD51.

As part of the cellular response to DNA damage, RAD51 and BRCA2 co-localize to damage-induced foci, which are believed to represent sites where DSB repair takes place $[17,35]$. Therefore, it is possible that the RAD51/ BRCA2 interaction prevents the constitutive binding of
RAD51 to undamaged DNA, and that the RAD51 bound by BRCA2 will be reserved for immediate localization to sites of DNA damage. If this is the case, then there has to be a change in the RAD51/BRCA2 interaction in response to DNA damage that allows RAD51 to bind DNA and initiate the formation of nucleoprotein filaments at the sites of DSBs. Such a change might involve DNAdamage-induced phosphorylation of either BRCA2 or RAD51. Mutations that affect the ability of BRCA2 to interact with RAD51, or cause defects in the relocalization of the BRCA2/RAD51 complex, would be expected to have severe effects on the normal efficiency of homologous recombination, potentially leading to gross chromosomal rearrangements and cell death.

Although RAD51 co-localizes with both BRCA1 and BRCA2 in S-phase nuclei and in cells treated with DNAdamaging agents, it is important to bear in mind that the majority of BRCA1 does not physically associate with BRCA2 and RAD51 $[17,18]$. Similarly, there appears to be a pool of RAD51 that fails to form complexes with BRCA2. Indeed, to our knowledge, a 'protein complex' that contains BRCA1, BRCA2 and RAD51 has yet to be isolated. Moreover, it should be emphasized that most of the proteins that have been shown to interact with BRCA1 fail to associate with BRCA2, and vice versa (Fig. 1). Such exclusivity supports the argument that these two tumour suppressors form distinct complexes in vivo, although it is also likely that these complexes may interact in terms of their repair functions. The co-localization of these proteins in nuclear foci only suggests that these two complexes are localized to potential repair sites; it does not necessarily mean that the three proteins interact directly and act in concert.

\section{Conclusion}

In summary, we suggest that BRCA1 and BRCA2 are capable of playing quite different roles, although it is undoubtedly also possible that complexes containing BRCA1 and BRCA2 may interact transiently to effect key reactions in DSB repair. It is hoped that further dissection of the functions of the novel partner proteins, present in complexes either with BRCA1 or BRCA2, will help to define the precise roles of BRCA1 and BRCA2 in DSB repair. Until then, the proposal that these important proteins influence the same or different steps of the DSB repair pathway will remain open to discussion and insightful experimentation.

\section{Acknowledgement}

Work in the laboratory of SCW is supported by the Imperial Cancer Research Fund. YL is a recipient of a postdoctoral fellowship from the American Cancer Society.

\section{References}

1. Rahman N, Stratton MR: The genetics of breast cancer susceptibility. Annu Rev Genet 1998, 32:95-121.

2. Eeles RA: Future possibilities in the prevention of breast cancer: Intervention strategies in BRCA1 and BRCA2 mutation 
carriers. Breast Cancer Res 2000 2:283-290.

3. Welcsh PL, King MC: BRCA1 and BRCA2 and the genetics of breast and ovarian cancer. Hum Mol Genet 2001, 10:705-713.

4. Ludwig T, Chapman DL, Papaioannou VE, Efstratiadis A: Targeted mutations of breast cancer susceptibility gene homologs in mice: lethal phenotypes of BRCA1, BRCA2, $B R C A 1 / B R C A 2, B R C A 1 / p 53$, and BRCA2/p53 null zygous embryos. Genes Dev 1997, 11:1226-1241.

5. Sharan SK, Morimatsu M, Albrecht U, Lim SS, Regel E, Dinh C, Sands A, Eichele G, Hasty P, Bradley A: Embryonic lethality and radiation hypersensitivity mediated by RAD51 in mice lacking BRCA2. Nature 1997, 386:804-810.

6. Suzuki A, Delapompa JL, Hakem R, Elia A, Yoshida R, Mo R, Nishina $\mathrm{H}$, Chuang T, Wakeham A, Itie A, Koo W, Billia P, Ho A, Fukumoto M, Hui CC, Mak TW: BRCA2 is required for embryonic cellular proliferation in the mouse. Genes Dev 1997, 11: 1242-1252.

7. Cortez D, Wang Y, Qin J, Elledge SJ: Requirement of ATMdependent phosphorylation of BRCA1 in the DNA damage response to double-strand breaks. Science 1999; 286:11621166.

8. Hohenstein $\mathrm{P}$, Kielman MF, Breukel $\mathrm{C}$, Bennett LM, Wiseman $\mathrm{R}$ Krimpenfort P, Cornelisse G, van Ommen GJ, Devilee P, Fodde R: A targeted mouse BRCA1 mutation removing the last BRCT repeat results in apoptosis and embryonic lethality at the headfold stage. Oncogene 2001, 20:2544-2550.

9. Yu VPCC, Köehler M, Steinlein C, Schmid M, Hanakahi LA, van Gool AJ, West SC, Venkitaraman AR: Gross chromosomal rearrangements and genetic exchange between non-homologous chromosomes following BRCA2 inactivation. Genes Dev 2000, 14:1400-1406.

10. Frankish $\mathrm{H}$ : BRCA1 has a pivotal role in repairing DNA. Lancet 2001, 357:1678-1678.

11. Patel KJ, Yu VPCC, Lee H, Corcoran A, Thistlethwaite FC, Evans MJ, Colledge WH, Friedman LS, Ponder BAJ, Venkitaraman AR: Involvement of BRCA2 in DNA repair. Mol Cell 1998, 1:347357.

12. Moynahan ME, Chiu JW, Koller BH, Jasin M: BRCA1 controls homology-directed DNA repair. Mol Cell 1999; 4:511-518.

13. Snouwaert JN, Gowen LC, Latour AM, Mohn AR, Xiao A, Di Biase $\mathrm{L}$, Koller $\mathrm{BH}$ : BRCA1 deficient embryonic stem cells display a decreased homologous recombination frequency and an increased frequency of non-homologous recombination that is corrected by expression of a BRCA1 transgene. Oncogene 1999, 18:7900-7907.

14. Moynahan ME, Pierce AJ, Jasin M: BRCA2 is required for homology-directed repair of chromosomal breaks. Mol Cell 2001, 7: 263-272.

15. Connor F, Bertwistle D, Mee PJ, Ross GM, Swift S, Grigorieva E, Tybulewicz VLJ, Ashworth A: Tumourigenesis and a DNA-repair defect in mice with a truncating BRCA2 mutation. Nature Genet 1997, 17:423-430.

16. Foray N, Randrianarison V, Marot D, Perricaudet M, Lenoir G, Feunteun J: Gamma rays induced death of human cells carrying mutations of BRCA1 or BRCA2. Oncogene 1999, 18:73347342.

17. Chen JJ, Silver DP, Walpita D, Cantor SB, Gazdar AF, Tomlinson G, Couch FJ, Weber BL, Ashley T, Livingston DM, Scully R: Stable interaction between the products of the BRCA1 and BRCA2 tumor suppressor genes in mitotic and meiotic cells. Mol Cell 1998, 2:317-328.

18. Wang Y, Cortez D, Yazdi P, Neff N, Elledge SJ, Qin J: BASC, a super complex of BRCA1-associated proteins involved in the recognition and repair of aberrant DNA structures. Genes Dev 2000, 14:927-939

19. Kerr $P$, Ashworth A: New complexities for BRCA1 and BRCA2. Curr Biol 2001, 11:R668-R676.

20. Deng CX, Brodie SG: Roles of BRCA1 and its interacting proteins. Bioessays 2000, 22:728-737.

21. Chen PL, Chen CF, Chen YM, Xiao J, Sharp ZD, Lee WH: The BRC repeats in BRCA2 are critical for RAD51 binding and resistance to methyl methanesulfonate treatment. Proc Nat Acad Sci USA 1998, 95:5287-5292.

22. Wu LC, Wang ZW, Tsan JT, Spillman MA, Phung A, Xu XL, Yang MC, Hwang LY, Bowcock AM, Baer R: Identification of a RING protein that can interact in vivo with the BRCA1 gene product. Nat Genet 1996, 14:430-440.
23. Hashizume R, Fukuda M, Maeda I, Nishikawa H, Oyake D, Yabuki Y, Ogata F, Ohta T: The RING heterodimer BRCA1-BARD1 is a ubiquitin ligase inactivated by a breast cancer-derived mutation. J Biol Chem 2001, 276:14537-14540.

24. Cantor SB, Bell DW, Ganesan S, Kass EM, Drapkin R, Grossman S, Wahrer DCR, Sgroi DC, Lane WS, Haber DA, Livingston DM: BACH1, a novel helicase-like protein, interacts directly with BRCA1 and contributes to its DNA repair function. Cell 2001, 105:149-160.

25. Zhang XD, Morera S, Bates PA, Whitehead PC, Coffer Al, Hainbucher K, Nash RA, Sternberg MJE, Lindahl T, Freemont PS Structure of an XRCC1 BRCT domain: a new protein-protein interaction module. EMBO J 1998, 17:6404-6411.

26. Ruffner $\mathrm{H}$, Joazeiro CAP, Hemmati $\mathrm{D}$, Hunter $T$, Verma IM: Cancer-predisposing mutations within the RING domain of BRCA1: loss of ubiquitin protein ligase activity and protection from radiation hypersensitivity. Proc Natl Acad Sci USA 2001 98:5134-5139.

27. Jensen DE, Proctor M, Marquis ST, Gardner HP, Ha SI, Chodosh LA, Ishov AM, Tommerup $N$, Vissing $H$, Sekido $Y$, Minna J, Borodovsky A, Schultz DC, Wilkinson KD, Maul GG, Barlev N, Berger SL, Prendergast GC, Rauscher FJ: BAP1: a novel ubiquitin hydrolase which binds to the BRCA1 RING finger and enhances BRCA1-mediated cell growth suppression. Oncogene 1998, 16:1097-1112.

28. Garcia-Higuera I, Taniguchi T, Ganesan S, Meyn MS, Timmers C, Hejna J, Grompe M, D'Andrea AD: Interaction of the Fanconi anemia proteins and BRCA1 in a common pathway. Mol Cell 2001, 7:249-262.

29. Paull TT, Gellert M: Direct DNA binding by BRCA1. Proc Nat/ Acad Sci USA 2001, 98:6086-6091.

30. Wang HC, Zeng ZC, Bui TA, DiBiase SJ, Qin W, Xia F, Powell $\mathrm{SN}$, lliakis G: Non-homologous end-joining of ionizing radiation-induced DNA double-stranded breaks in human tumor cells deficient in BRCA1 or BRCA2. Cancer Res 2001, 61:270277.

31. Baumann P, West SC: Role of the human RAD51 protein in homologous recombination and double-stranded break repair. Trends Biochem Sci 1998, 23:247-251.

32. Wong AKC, Pero R, Ormonde PA, Tavtigian SV, Bartel PL: RAD51 interacts with the evolutionarily conserved BRC motifs in the human breast cancer susceptibility gene BRCA2. J Biol Chem 1997, 272:31941-31944.

33. Davies AA, Masson J-Y, Mcllwraith MJ, Stasiak AZ, Stasiak A Venkitaraman AR, West SC: Role of BRCA2 in control of the RAD51 recombination and DNA repair protein. Mol Cell 2001, 7:273-282.

34. Chen CF, Chen PL, Zhong Q, Sharp ZD, Lee WH: Expression of BRC repeats in breast cancer cells disrupts the BRCA2RAD51 complex and leads to radiation hypersensitivity and loss of G(2)/M checkpoint control. J Biol Chem 1999, 274: 32931-32935.

35. Scully R, Chen J, Plug A, Xiao Y, Weaver D, Feunteun J, Ashley T, Livingston DM: Association of BRCA1 with RAD51 in mitotic and meiotic cells. Cell 1997, 88:265-275. 